\title{
Effects of sildenafil on sperm DNA structure
}

\section{Proceeding}

We evaluated the effects of sildenafil on sperm motility and sperm DNA fragmentation index (DFI). A semen sample was collected from each of 20 men (group A) selected from a general population of men visiting a urology outpatient clinic. After a swim up procedure, motile spermatozoa populations were collected from each sample. Then two $1 \mathrm{ml}$-aliquots (C and EXP aliquots) containing washed spermatozoa suspended in a culture medium were prepared from each of the above 20 men. Sildenafil was added to EXP aliquots at a final concentration equal to 0.67 microM. C aliquots served as control aliquots. Each pair of aliquots was incubated at $37^{\circ} \mathrm{C}$ under $5 \%$ carbon dioxide for 8 hours. At the end of the incubation period the \% motile sperms (\%MS) and the DFI as measured with the sperm chromatin structure assay were evaluated (Asian J Androl 2011,13:69).

Within group A, the mean value of the DFI was significantly larger in Exp aliquots (mean \pm SD:29.17 $\pm 11.67 \%$ ) than in $\mathrm{C}$ aliquots $(22.45 \pm 11.17 \%)$ (Wilcoxon test for paired observations; $\mathrm{P}<0.05)$. On the other hand, within the group A, there were no significant differences in the mean value of \%MS between Exp aliquots and $\mathrm{C}$ aliquots. It may be suggested that elevation of the second messenger cGMP level due to inhibition of PDE5 by sildenafil activates a nuclear cGMPdependent protein kinase PKG with an overall detrimental effect on

\author{
Volume 5 Issue 4 - 2017 \\ I Giakoumakis, I Giannakis, D Baltogiannis, \\ L Lazaros, D Daphnis, F Dimitriadis, A \\ Takenaka, N Sofikitis \\ Mediterranean Fertility Center \& Genetic Services, Greece
}

Correspondence: loannis Giakoumakis, OB-GYN, Clinical Director of Mediterranean Fertility Institute (Surrogacy, Egg Donation, In Vitro Fertilization Program), Alexandra General Hospital in Athens, 24 Zimvrakakidon St., Chania, Greece, Email igiakoumakis@ivfgreece.com

Received: July 10, 2017 | Published: July 10, 2017

sperm chromatin structure. Alternatively we may hypothesize that the effect of sildenafil on sperm DNA is due to the formation of hydrogen bonds between the $\mathrm{C}=\mathrm{O}$ groups of the molecule of sildenafil and the $\mathrm{NH}_{2}$ group in the guanine moiety of the DNA. The latter hypothesis is strongly supported by previous research efforts indicating a similar mechanism responsible for the interaction between sildenafil with salmon sperm DNA (Biosensors and Bioelectronics 22,2007,2471).

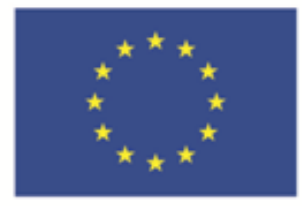

European Union European Social Fund

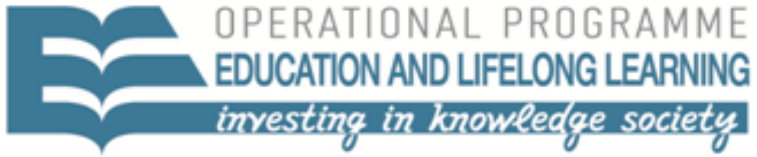

MINISTRY OF EDUCATION \& RELIGIOUS AFFAIRS $M A N A G I N G$ A U T H O R I T Y

Co-financed by Greece and the European Union

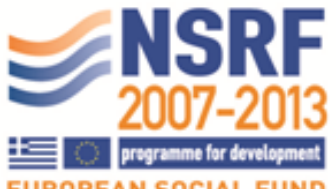

EUROPEAN SOCIAL FUND
This research has been co-financed by the European Union (European Social Fund-ESF) and Greek national funds through the Operational Program "Education and Lifelong Learning" of the National Strategic Reference Framework (NSRF)-Research Funding Program: Heracleitus II. Investing in knowledge society through the European Social Fund.

\section{Acknowledgements}

None.

\section{Conflict of interest}

The author declares no conflict of interest. 